\title{
Assessing similarities and disparities in the skin microbiota between wild and laboratory populations of house mice
}

\author{
Meriem Belheouane ${ }^{1,2} \cdot$ Marie Vallier $\mathbb{D}^{1,2} \cdot$ Aleksa Čepić ${ }^{1,2} \cdot$ Cecilia J. Chung ${ }^{1,2} \cdot$ Saleh Ibrahim ${ }^{3} \cdot$ John F. Baines $\mathbb{D}^{1,2}$
}

Received: 25 June 2019 / Revised: 15 May 2020 / Accepted: 22 May 2020 / Published online: 9 June 2020

(c) The Author(s) 2020. This article is published with open access

\begin{abstract}
The house mouse is a key model organism in skin research including host-microbiota interactions, yet little is known about the skin microbiota of free-living mice. It is similarly unclear how closely laboratory mice, which typically live under exceptionally hygienic conditions, resemble the ancestral state of microbial variation in the wild. In this study, we sampled an area spanning $270 \mathrm{~km}^{2}$ in south-west France and collected 203 wild Mus musculus domesticus. We profiled the ear skin microbiota on standing and active communities (DNA-based and RNA-based 16 rRNA gene sequencing, respectively), and compared multiple community aspects between wild-caught and laboratory-reared mice kept in distinct facilities. Compared to lab mice, we reveal the skin microbiota of wild mice on the one hand to be unique in their composition within the Staphylococcus genus, with a majority of sequences most closely matching known novobiocin-resistant species, and display evidence of a rare biosphere. On the other hand, despite drastic disparities between natural and laboratory environments, we find that shared taxa nonetheless make up the majority of the core skin microbiota of both wild- and laboratory skin communities, suggesting that mammalian skin is a highly specialized habitat capable of strong selection from available species pools. Finally, the influence of environmental factors suggests RNA-based profiling as a preferred method to reduce environmental noise.
\end{abstract}

\section{Introduction}

The skin serves critical functions as a physical and immunological barrier, but is also a dynamic ecosystem inhabited by diverse microbial symbionts. This ecosystem is influenced by fundamental processes of community assembly including dispersal, local diversification, environmental selection, and ecological drift [1-3]. Understanding the

Supplementary information The online version of this article (https:// doi.org/10.1038/s41396-020-0690-7) contains supplementary material, which is available to authorized users.

John F. Baines

baines@evolbio.mpg.de

1 Max Planck Institute for Evolutionary Biology, AugustThienemann-Str. 2, 24306 Plön, Germany

2 Institute for Experimental Medicine, Kiel University, ArnoldHeller-Str. 3, 24105 Kiel, Germany

3 Lübeck Institute of Experimental Dermatology, University of Lübeck, Lübeck, Germany relative contribution of these forces remains challenging and largely unsolved [4, 5].

Natural populations are valuable resources for investigating principles of community assembly and potential selective pressures on the host. Free-living individuals are confronted with heterogeneous environments comprised of diverse species pools and are regularly exposed to a wide range of pathogens, underscoring the need for efficient immune strategies to maintain skin barrier function and inflammatory homeostasis. Several recent studies [6-8], revealed that wild mice reflect the immune responses of adult humans far better than laboratory mice, suggesting that wild mice may be valuable to inspect aspects of the hygiene hypothesis, immune functioning and potential treatment of autoimmune and inflammatory disorders.

Previous efforts explored the composition of skinassociated microbiota and surrounding environments across different species living in the same habitat, and addressed the role of resident bacteria on host survival in natural populations [9-14]. These pioneer studies revealed two key findings: (i) host species identity is the strongest predictor of community composition, (ii) the impact of the surrounding environments on the microbiota composition 
are limited. Importantly, signatures of phylosymbiosis in the mammalian skin microbiota serve as first evidence of coevolutionary processes [14], which potentially carry fitness consequences such as those observed for the gut microbiota in deer mice [15]. Indeed, several studies in amphibians demonstrated resident skin bacteria to play a critical role in resistance to fungal infection, and thus directly contribute to host fitness in nature [16-19].

The house mouse is a key model organism for microbiome research and is intensively studied [20-23]. Although several studies examined the gut microbiota of free-living mice [24-28] or the skin microbiota of other mammals [14, 29-31] still nothing is known about the normal range of variation of skin microbiota in wild house mice, nor how closely the microbial communities of laboratory mice, which are housed under controlled conditions and experience little to no external stimuli, reflect the natural state of microbial composition in the wild.

In this study we conducted fieldwork and sampled 203 wild house mice over an area of approximately $270 \mathrm{~km}^{2}$. We profiled the ear skin microbiota using 16S rRNA gene sequencing based on both extracted DNA-material and RNA-material (standing and active communities, respectively), and thoroughly compared multiple community aspects between wild-caught and three populations of laboratory-reared mice kept in different facilities. We reveal for the first time that the skin microbiota of wild mice is dominated by Staphylococcus genus, whose identity most closely matches known novobiocin-resistant species and displays an excess of rare taxa, but that community membership otherwise substantially overlaps between wild and laboratory populations. Moreover, we identify important structural disparities across mouse populations, and detect a pattern of similarity decay in community composition with geographic distance in the wild.

\section{Materials and methods}

\section{Wild mouse collection and tissue sampling}

In September and October of 2013, 203 wild-house mice were collected from 34 distinct farms and stables that were randomly chosen around the southwestern French commune of Espelette. Mean pairwise distance between sampling sites was $10 \mathrm{~km}$, and standard deviation (SD) $6 \mathrm{~km}$. For each mouse, the following parameters were recorded: sex, bodyweight, tail length, body length, female pregnancy status, and farm/stable animal breeding type. Trapped mice were first transported within $2 \mathrm{~h}$ to a common location for euthanization with $\mathrm{CO}_{2}$ followed by dissection. Dissections were performed with care to avoid cross contamination, whereby ear biopsies [32] only came in contact with instruments that were freshly cleaned $(70 \%$ ethanol, followed by RNase AWAY) and heat-sterilized prior to each dissection. Two distinct pieces of the left ear were sampled from each mouse for population structure analysis and $16 \mathrm{~S}$ rRNA gene profiling, respectively. Tissues were stored immediately at $-20^{\circ} \mathrm{C}$ and then shipped on dry ice to the laboratory, where they were stored at $-80^{\circ} \mathrm{C}$ until processing.

\section{Nucleic acid extraction}

For population genetic analysis (D-loop and microsatellites), DNA was extracted using the DNeasy Blood and Tissue Kit (Qiagen) following the manufacturer's instructions. DNA and RNA for 16S rRNA gene sequencing were extracted from the tip of the left ear using the AllPrep DNA/ RNA 96 Kit (Qiagen) along with blank negative extraction controls. Samples were homogenized through bead beating using the FastPrep ${ }^{\circledR}-96$ (MP Biomedicals) $2 \times 45$ s at speed 4.0 , then held for $2 \mathrm{~h}$ at room temperature in buffer RLT to increase nucleic acids yield. RNA was treated with DNase (Qiagen) for $15 \mathrm{~min}$, twice. Complementary DNA (cDNA) synthesis was performed using a High-Capacity cDNA Reverse Transcription Kit (AB Applied Biosystems). RNA purity was checked by a negative Reverse Transcriptase control (without transcriptase) PCR reaction and agarose gel electrophoresis.

\section{Mitochondrial D-loop sequencing and haplogroup analysis}

An $885 \mathrm{bp}$ portion of the mitochondrial D-loop was sequenced as described by Prager et al. [33] Sequences edited in Geneious (v.7.0) [34] were aligned in MEGA 5 [35] to Mus musculus domesticus, Mus spretus, and Mus spicilegus reference sequences (GenBank Accessions: AM182648, U47539, U47536, respectively) and sequences from Linnenbrink et al. [24]. A NeighbourNet network was constructed with Huson and Bryant's hypothesis-poor algorithm using the SplitsTree package (v.4.10) [36]. Individual wild mice were clustered into haplogroups previously defined by Bonhomme et al. [37]. p-distance, which represents the proportion $(p)$ of different nucleotide sites between two compared sequences, was calculated in MEGA 5 using default parameters.

\section{Microsatellite typing and population structure analysis}

We typed 18 unlinked neutral autosomal microsatellites as described by Thomas et al. [38] and Hardouin et al. [39] in Geneious (v.7.0). Allele tables were then transferred, and analyzed in STRUCTURE (2.3.4) [40-42] to infer the 
population structure (See Supplementary Methods). Mice with $\geq 80 \%$ ancestry assigned to a given cluster were considered to be reliably assigned to that cluster, and referred to as "non-admixed", whereas the remainder were classified as "admixed", but assigned to the cluster making up the largest portion of their ancestry. Cavalli-Sforza distance (CAS), an absolute measure of genetic distance [43], was calculated using GenoDive (v.2.0) [44].

\section{$16 S$ rRNA gene sequencing and processing in wild mice}

The hypervariable V1-V2 region of the bacterial 16S rRNA gene was amplified using $27 \mathrm{~F}$ and $338 \mathrm{R}$ primers following a dual indexing approach on the Miseq Illumina platform as described in Supplementary Methods. For sequence processing, no mismatch in the barcode was allowed while demultiplexing (Casava, Illumina). Forward and reverse reads were filtered in $\mathrm{R}$ (v.3.6.1) using the "DADA2" package (v.1.14) [45] as follows: truncated at the first base for which the quality score dropped below $Q=2$, no ambiguous nucleotides were allowed, maximum expected errors maxEE were 2 and 5, and minimum length of truncated reads were 250 and $200 \mathrm{bp}$ for forward and reverse reads, respectively. Sequence reads were then subjected to the de-noising algorithm with the "pool=pseudo" option to increase sensitivity to low frequency sequence variants. Denoised forward and reverse reads were merged with a minimum identical overlap of $20 \mathrm{bp}$, amplicon sequence variants (ASVs) were inferred, and chimeric ASV sequences were removed using the de novo consensus method. Afterwards, ASVs whose length exceeded $350 \mathrm{bp}$ were excluded, and ASV taxonomy was assigned from the phylum to genus level using the Silva reference database (release 132) [46] with bootstrap confidence $\operatorname{minBoot}=80$. ASVs assigned to Eukaryota, unclassified kingdom or mitochondria were excluded.

\section{Identification of contaminant ASVs}

To identify potential contaminant ASVs, we applied the statistical classification procedure implemented in the "Decontam" (v.1.6.0) R package [47]. First, the DNA and RNA concentration of each extracted skin sample was measured, including all negative extraction controls, using the fluorescent Qubit dsDNA and the fluorescent Qubit RNA broad range assay kits (life technologies) for DNA and RNA, respectively. The concentrations are provided in Supplementary Table 1 . Specifically, the frequency method was used, which assumes that sequences from contaminating taxa are likely to inversely correlate with sample DNA and RNA concentration. Those negative extraction controls that displayed any PCR amplification (4/4 and 3/4 controls for DNA and RNA, respectively) and whose total processed reads equaled or exceeded 40 were included (4/4 and 2/4 controls for DNA and RNA, respectively). With a probability threshold of 0.1 we identified contaminant ASVs distinctly in DNA and RNA datasets, and excluded the combined defined contaminants (204 ASVs) for subsequent analyses.

\section{Normalization of sequencing depth across samples}

We normalized the read distribution to an equal sequencing depth across samples to (i) reduce biases in subsequent ecological analyses [48], and (ii) further detect inaccurate/ suspicious ASVs that may result from sequencing and/or processing artifacts that could alter diversity measures based on taxon presence/absence. Accordingly, we randomly drew 4000 reads (close to the minimum depth in the dataset of 4321 reads) 1000 times independently for each sample. Afterwards the distribution of ASV frequency across the 1000 independent draws was inspected, and ASVs whose frequency across the 1000 draws equaled or exceeded the $10 \%$ quantile of frequency distribution of all ASVs were selected, and a final sample of 4000 reads was drawn exclusively from the selected ASVs. Excluded ASVs represent between 0.06 and $2.7 \%$ of the initial nonnormalized sequences across samples. After normalization, we included 30,361 ASVs jointly in the DNA and RNA datasets (average $250 \mathrm{ASVs}, \mathrm{SD}=179$ )

\section{Comparison of skin microbiota between wild and laboratory-reared mice}

In order to compare wild and laboratory mice, we included three additional groups of laboratory mice reared in distinct facilities: (i) an advanced intercross between $M . m$. domesticus and M. m. castaneus strains described in Belheouane et al. [49] $(n=225)$, reared at the University of Luebeck, Germany, hereafter referred to as HL-Lab, (ii) a mixed collection of wild-derived M. m. domesticus $(n=29)$ that includes $\mathrm{WSB} / \mathrm{EiJ}(n=9)$, wild-derived individuals from Cologne/Bonn, Germany (CB) $(n=11)$, and the Massif Central, France (MC) $(n=9)$ that were bred under laboratory conditions following an outbreeding scheme for nearly a decade, hereafter referred to as MPI-Lab, and (iii) the C57BL/6J strain $(n=13)$. MPI-Lab and C57BL/6J mice were reared at the Max Planck Institute for Evolutionary Biology, Ploen, Germany. The handling and sacrifice of wild and lab (see below) mice was conducted according to the German animal welfare law (Tierschutzgesetz) and Federation of European Laboratory Animal Science Associations guidelines. All mice were sacrificed with $\mathrm{CO}_{2}$ followed by cervical dislocation. Tissue sampling for scientific purposes was performed according to the German animal 
welfare law (Permit V 312-72241.123-34). Ear skin was sampled, processed, and 16S rRNA sequences were generated as described above.

Accordingly, we merged the 16S rRNA gene sequence datasets of the wild-caught $(n=203)$ and laboratory populations HL-Lab $(n=225)$, MPI-Lab $(n=29)$, and C57BL/ $6 \mathrm{~J}(n=13)$. Sequence processing and the identification of contaminant ASVs was performed as described above. In total, we included negative extraction controls for every individual extraction round $(n=21$ each for DNA and RNA, i.e., $n=42$ total). Of these, 18 for DNA and 19 for RNA yielded detectable amplification on an agarose gel using Image Lab Software (Bio-Rad) and were included in sequencing libraries. Of those negative extraction controls that were sequenced, 17 for DNA-based and 10 for RNAbased libraries retained a sufficient number of sequencing reads after processing ( $\geq 40$ reads; median of 3721 and 3158 DNA and RNA, respectively for negative extraction controls compared to 12,215 and 19,716 DNA and RNA, respectively for real samples) to be included in Decontam analysis. This resulted in the removal of 314 ASVs. We normalized sequencing depth across samples to 2000 reads per sample as described for the wild mice, resulting in the exclusion of ASVs that represent from 3.69 to $0.004 \%$ of non-normalized reads. We detected 36,353 ASVs in the entire DNA and RNA datasets (average 165 ASVs, SD: 104).

All statistical analyses were performed in R (v.3.6.1) (R Core Team, 2015). Comparison of main taxa across groups was performed using Wilcoxon tests, and $p$ values were corrected for multiple testing according to Benjamini and Hochberg [50]. Indicator species analysis was applied in the "indicspecies" R package (v.1.7.9) [51] using the "r.g" function [52] and $10^{5}$ permutations. Random Forest classification and regression analyses were carried out using the "randomForest" package [53] (4.16-14) with $10^{6}$ trees and "mtry $=13$ " for core DNA and RNA genera and "mtry $=5$ " for core Staphylococcus ASVs. Alpha diversity indices and principal coordinates analyses of Bray-Curtis and Jaccard indices were carried out with the "vegan" package using functions "diversity", "estimateR", and "cmdscale".

\section{Identification of Staphylococcus and Streptomyces species}

To determine species-level taxonomy of the Staphylococcus and Streptomyces ASVs, we selected samples (DNA) that harbored the highest absolute diversity of traits and amplified a longer portion of the $16 \mathrm{~S}$ rRNA gene in wild and HLLab individuals using genus-specific primers (see Supplementary Methods). PCR products were cloned using the CloneJet PCR kit from ThermoScientific and One Shot TOP10 Chemically Competent E. coli from Invitrogen, followed by Sanger sequencing. Taxonomy of trimmed clone sequences (approx. 800 and $500 \mathrm{bp}$ for Staphylococcus and Streptomyces, respectively) was defined in RDP match (v.16) [54] based on the highest seqmatch score (S_ab). When delta $S_{-}$ab values were lower than 0.05 , multiple matches were reported. Subsequently, sequences of clones, ASV representatives, and outgroups (Bacillus luciferensis "AJ419629.1" for Staphylococcus and Kitasatospora kifunensis "AJ781341.1" for Streptomyces) were aligned using the Geneious algorithm, and ASVs were matched to clones based on the highest alignment identity score (percentage of identical bases).

\section{High-throughput sequencing of the Staphylococcus tuf gene}

To improve the taxonomic classification of Staphylococcus species, we generated partial sequences of the tuf gene in a subset of 53 wild (from all 34 farms, 1-4 mice per farm), 41 from HL-Lab, 18 MPI-Lab, and 6 C57BL/6J DNA samples. Additionally, we included two replicates of a microbial community standard (ZymoBIOMICS) containing S. aureus and the negative extraction controls $(n=17)$. We followed a two-step PCR strategy using Staphylococcus-specific primers and sequenced amplicons on the Miseq Illumina platform. Sequence processing and the definition of ASVs were performed as describe above. Representative ASV sequences were aligned to a Staphylococcus tuf gene database, which includes 36 sequences and 1 sub-species used in McMurray et al. [55], and with a sequence from the closest genus to Staphylococcus [56] i.e., Macrococcus canis (accession number: KM45013) as an outgroup (See Supplementary Methods and Supplementary Table 10). Negative extraction controls were included and treated in the same manner as for the 16S rRNA gene sequencing. A single negative extraction control out of 21 displayed any amplification, but after sequencing processing contained only a single sequence that did not belong to Staphylococcus.

\section{Analysis of sources of variation in skin microbiota composition in wild mice}

To further characterize skin microbiota community of the wild-caught mice, we calculated alpha and beta diversity indices based on ASV distribution in the "vegan" package (v.2.5.6) as described above [57]. Representative ASV sequences were aligned in DECIPHER (v.2.14) [58], a distance matrix was calculated in "phangorn" (2.5.5) [59] and a Neighbor-Joining tree was inferred. Phylogenetic diversity (PD) was calculated according to Faith et al. [60] using the "picante" package (v.1.8) [61]. Unweighted and weighted UniFrac metrics [62, 63] based on ASVs were 
calculated using the "Fast UniFrac" algorithm in the "Phyloseq" package (v.1.30) [64]. The influence of sampling location and host features (sex, weight, proportion of body to tail length, pregnancy, and body mass index) on the composition of standing and active bacterial communities was assessed through a linear mixed effects approach using restricted maximum likelihood (REML) in the "Ime4" $\mathrm{R}$ package (v1.1-21) [65]. Furthermore, the impact of distance between sampling locations on community structure and diversity was determined (See Supplementary Methods).

\section{Results}

To gain critical knowledge on the microbial communities inhabiting the ear skin of wild house mouse, we analyzed 203 M. m. domesticus individuals coming from 34 distinct locations around the southwestern French commune of Espelette. Further, we recently determined that community profiling based on 16S rRNA gene transcripts may better reflect true residents and underlying interactions with the host [49] due to the skin low microbial biomass and potential for environmental noise. Thus, we performed $16 \mathrm{~S}$ rRNA gene sequencing using both bacterial genomic DNA and RNA reverse transcribed into cDNA as templates, which we refer to as the "standing" and "active" communities, respectively. To compare these data to skin microbial composition typically observed in a laboratory environment, we included three different groups of laboratory mice reared in distinct facilities: HL-Lab [49], MPI-Lab, and C57BL/6J (Table 1, Supplementary Table 1).

\section{Skin microbiota composition in wild and laboratory mice}

First, we assessed the relative abundances of the five most abundant phyla (defined as one of the five most abundant taxa in at least two of the four groups, comprising $90.57 \%$, $\mathrm{SD}=14.09 \%$, and $82 \%, \mathrm{SD}=12.32 \%$, of the total abundance for DNA and RNA datasets, respectively), and five most abundant genera (comprising $38 \%, \mathrm{SD}=18.47 \%$, and $30 \%, \mathrm{SD}=14.31 \%$ of the total abundance for DNA and RNA datasets, respectively). This reveals Firmicutes to be the most abundant phylum in wild, MPI-Lab, and C57BL/ $6 \mathrm{~J}$ mice on the DNA level, which is significantly lower in HL-Lab mice, whereas Proteobacteria is more abundant in C57BL/6J.

Of note, a clear and significantly higher Actinobacteria abundance is also a shared aspect of the wild and C57BL/6J mice at the DNA level, whereas on the RNA level Actinobacteria remains highly abundant in the wild and Firmicutes dominates the C57BL/6J community (Fig. 1a, Supplementary Table 2). Among the genera detected at the

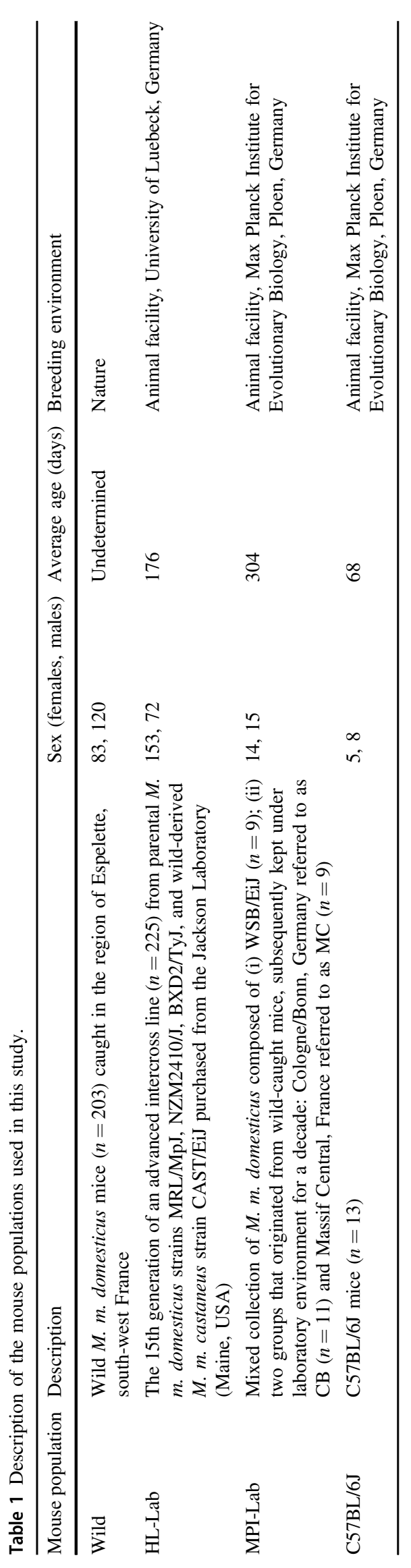



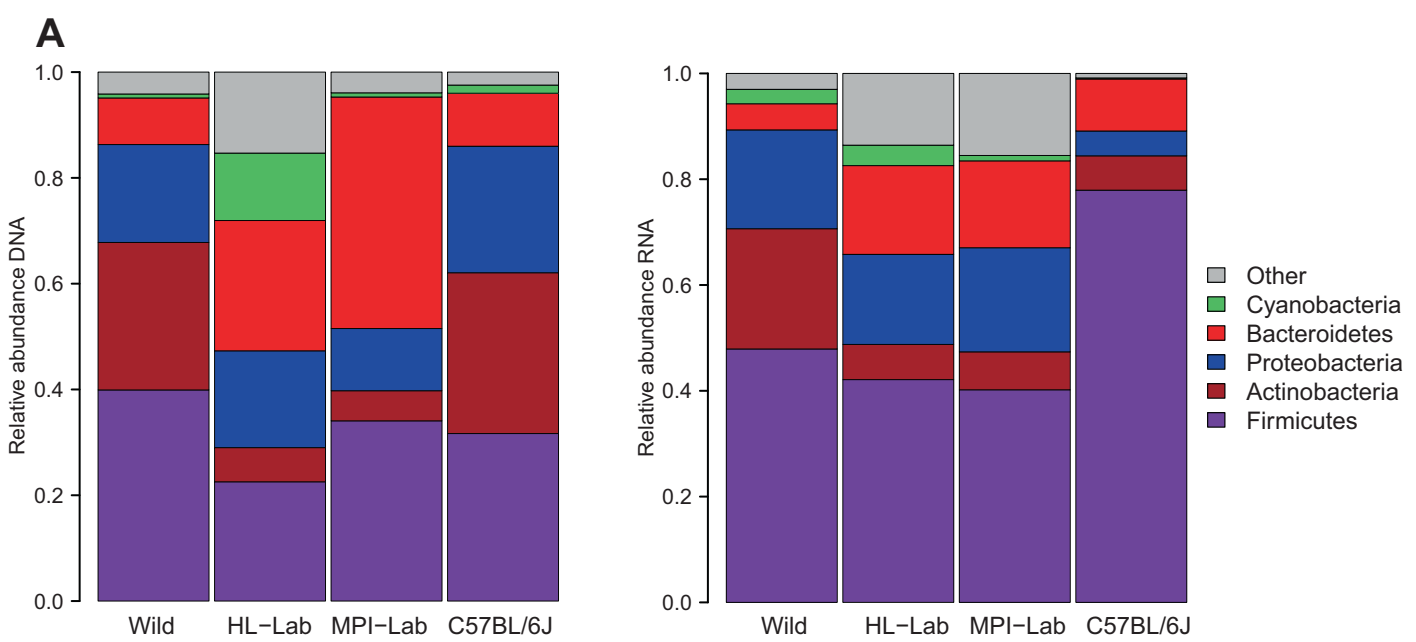

\section{B}
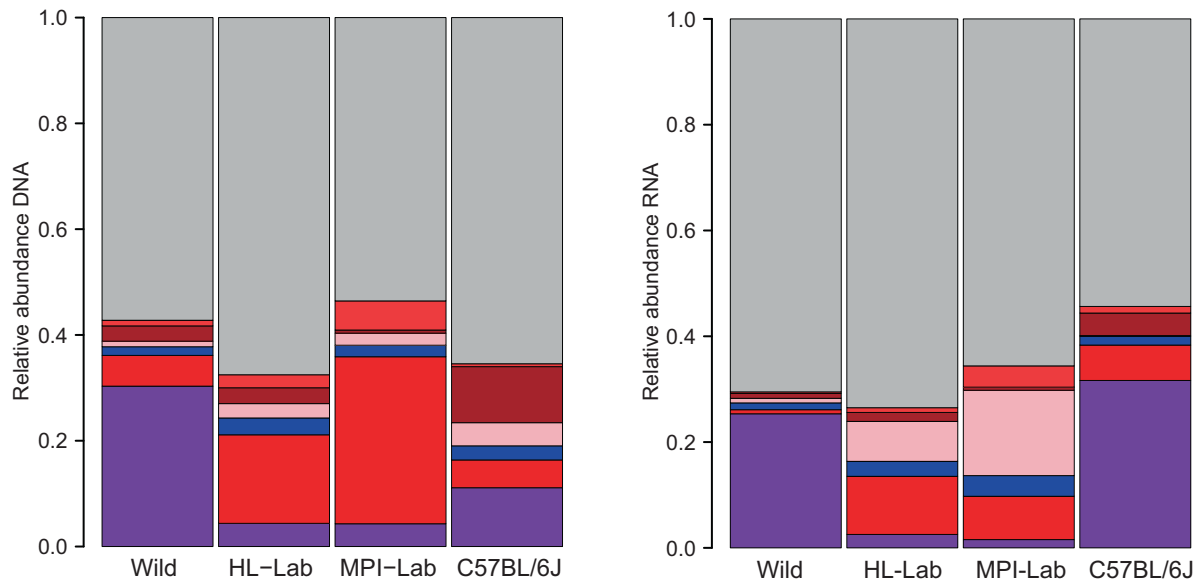
Fig. 2 Alpha diversity indices.

Shannon and Chao1 indices across mouse populations in standing (DNA-based) (a) and active (RNA-based) (b) communities. Pairwise Wilcoxon tests are reported in Supplementary Table 2 .
Fig. 3 Unique and shared core genera in mouse populations. Standing (DNA-based) (a) and active (RNA-based) (b) communities. Summary statistics of core and unique genera are reported in Supplementary Table 2.
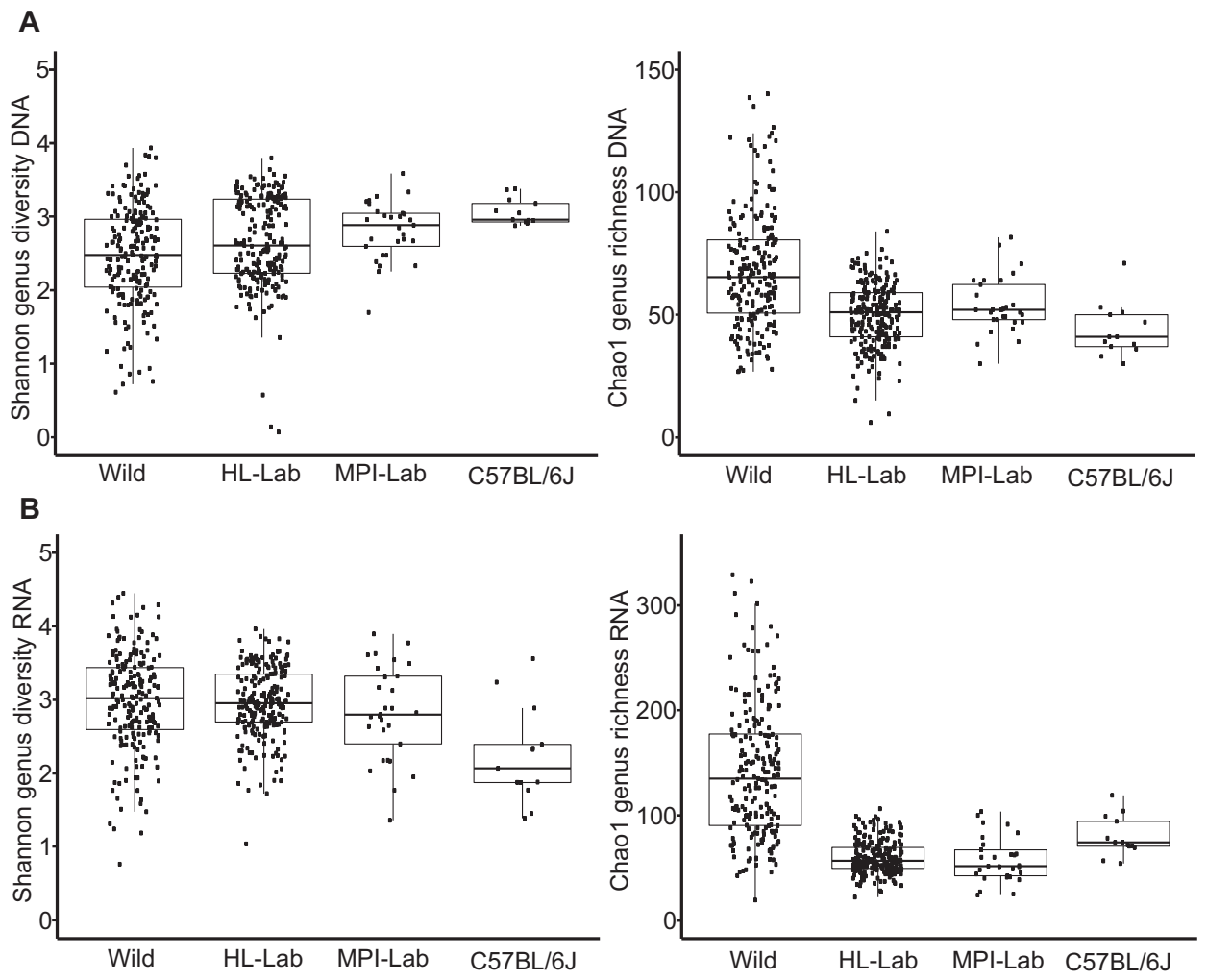

A

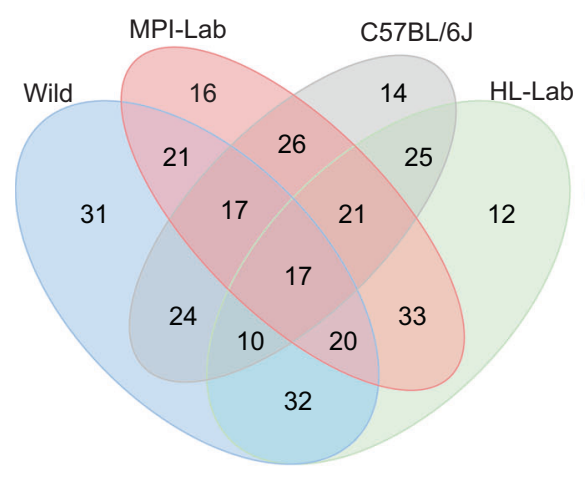

B

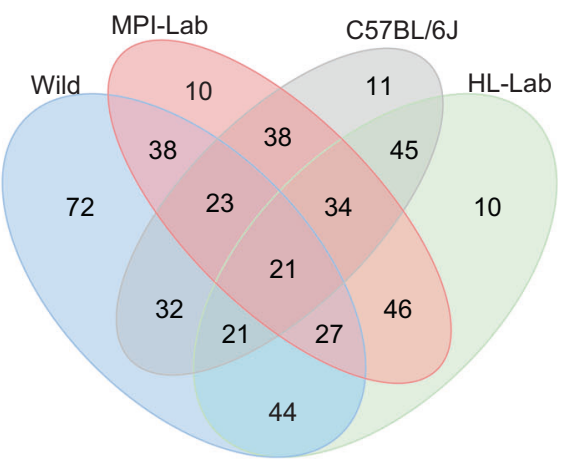

accurate picture of taxa inhabiting each mouse population, we limited the analyses to core phyla and genera that we defined within each population's DNA and RNA datasets as present in at least $25 \%$ of the individuals. Core taxa represent approximately 99 and $90 \%$ of all phyla and genera, respectively, detected within mouse populations (Supplementary Table 2). In all cases, wild mice display by far the largest number of unique genera, which is consistent with the observations of genus-level richness.

Despite the higher number of taxa unique to wild mice, the smaller number of taxa shared among all four groups ( 5 and 6 phyla and 17 and 21 genera in standing and active communities, respectively) comprises the highest proportion of the core abundance within communities.
Specifically, shared genera at the DNA level represent on average from $58 \%$ in wild to $78 \%$ in MPI-Lab. Unique genera make up the second largest proportions in wild (27\%) and C57BL/6J (19\%), whereas unique genera represent comparatively minor fractions in HL-LAB (5\%) and MPI-LAB (9\%). Shared core genera based on RNA represent between $48 \%$ in wild to $65 \%$ in C57BL/6J. Notably, unique RNA genera represent minor fractions in all laboratory groups (from $1 \%$ in $\mathrm{C} 57 \mathrm{BL} / 6 \mathrm{~J}$ to $9 \%$ in $\mathrm{HL}-$ $\mathrm{Lab}$ ), while in the wild the unique fraction remains the second largest (32\%).

To thoroughly assess beta diversity, we analyzed the Bray-Curtis and Jaccard indices, which are based on weighted taxon abundances and taxon presence/absence, 
A

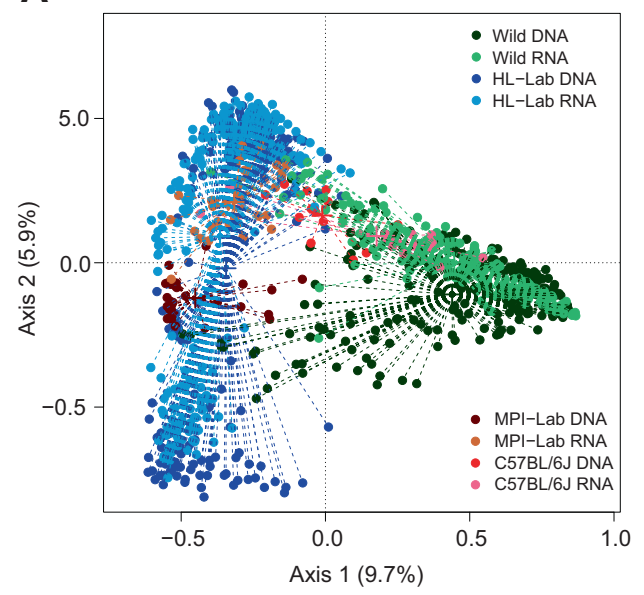

B

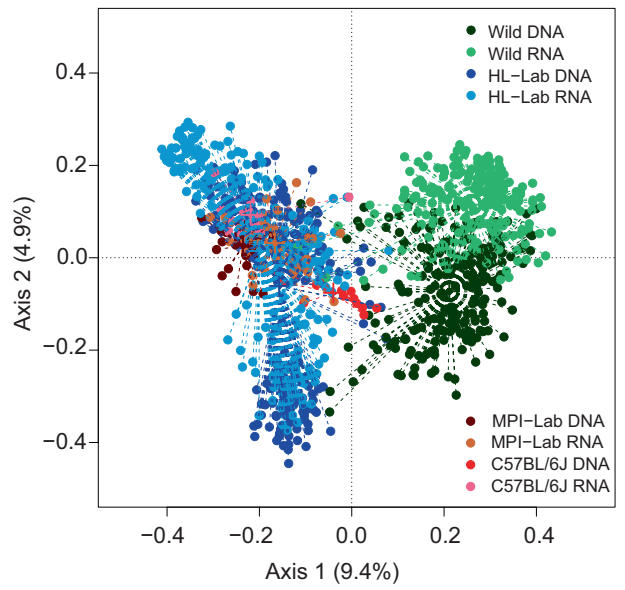

Fig. 4 Beta diversity indices. Unconstrained principal coordinates analysis (PCoA) of Bray-Curtis (a) and Jaccard (b) indices (genus-level) in mouse populations in standing (DNA-based) and active (RNA-based) communities. Goodness of fit of mouse origin: Bray-Curtis axes, $R^{2}=0.49$, $p=10^{-5}$, Jaccard: $R^{2}=0.62, p=10^{-5}$, based on $10^{5}$ permutations. "+" centroid of the cluster.

respectively (Fig. 4 and Supplementary Fig. 2). Principal coordinates analysis of Bray-Curtis index reveals a large effect of sample origin, with the first, second, and third axes being significant (Fig. 4a). This effect is larger than that of profiling type (i.e., standing versus active communities). Interestingly, C57BL/6J mice display an intermediate pattern of community structure between the wild, HL-Lab, and MPI-Lab mice. Analysis based on the Jaccard index, on the other hand, reveals a more substantial distinction between the wild and three laboratory mouse groups, with an additionally more pronounced difference between the standing and active communities of the wild mice (Fig. 4b). Moreover, we inspected the influence of additional features including location (farm, familylcage) and sex, and find a significant influence of mouse location and sex (permanova adonis, Bray-Curtis: mouse origin $R^{2}=0.21, p=10^{-5}$, location $R^{2}=0.15, p=10^{-5}$, sex $R^{2}=0.004, p=10^{-5}$; Jaccard: mouse origin $R^{2}=0.13, p=10^{-5}$, location $R^{2}=$ $0.14, p=10^{-5}$, sex $R^{2}=0.002, p=10^{-5}$, based on $10^{5}$ permutations).

\section{Indicator species and random forest classification analyses}

To identify individual taxa contributing to patterns of beta diversity, we first performed indicator species analysis based on the joint core genera (i.e., the sum of all those present in $\geq 25 \%$ of the mice in any given group) relative abundance and presence/absence in the standing and active communities (Supplementary Table 3). Analysis based on relative abundance in the standing communities identifies Staphylococcus, Streptomyces, and Brevibacterium as strong indicators for wild-caught individuals. Indicators of laboratory mice include Cutibacterium and
Corynebacterium_1, which are strongly associated to C57BL/6J, and several Clostridiales and Bacteroidetes genera associated to MPI-Lab.

In the active communities, Staphylococcus is a strong common indicator of wild and C57BL/6J, whereas, Burkholderia and Streptococcus become significant indicators of MPI-Lab mice. These results are clearly in line with the compositional contrasts described above. Interestingly, presence/absence analysis reveals numerous genera belonging to Actinobacteria, with Streptomyces showing the strongest association to wild mice.

To further assess the discriminating power of the core genera, we performed random forest classification analyses. We find that core DNA $(n=133)$ and RNA $(n=$ 191) genera accurately classify all individuals to their origin (OOB estimate of error rate: 1.7\%, mean classification accuracy across groups 100\%) (Supplementary Fig. 3). Moreover, we inspected the mean decrease accuracy component across genera and find that several Actinobacteria taxa including Streptomyces, Brevibacterium, and Nocardiopsis along with Staphylococcus are crucial to the accuracy of the classification (Supplementary Table 3 ).

Given the interesting patterns of Staphylococcus abundance across mouse groups, we additionally performed the analyses based on core Staphylococcus ASVs $(n=33)$. First, indicator analyses find most ASVs to be strongly associated to wild mice, and fewer with laboratory mice. Specifically, ASV_2, ASV_3, and ASV_4 are associated to wild mice, while ASV_1 and ASV_17 indicate C57BL/6J, and ASV_19 and ASV_77 jointly indicate HL-Lab and MPI-Lab (Supplementary Table 3). Random Forest analyses correctly classifies wild individuals, whereas $15 / 29$ to 21/29 MPI-Lab, and 2/13 C57BL/6J are assigned to 
HL-Lab (OOB estimate of error rate: 9.79 and $7.23 \%$, mean classification accuracy across groups 96.38 and $94.25 \%$ in DNA and RNA, respectively). Importance components find ASV_2, ASV_3, ASV_4, ASV_19, and ASV_11 as most crucial to classification (Supplementary Table 3). These results importantly show that Staphylococcus core ASVs are sufficient to accurately discriminate between wild and laboratory mice (Supplementary Fig. 4)

\section{Diversity of Staphylococcus and Streptomyces in wild and laboratory mice}

Given the notably higher abundance of Staphylococcus as a distinguishing feature of the wild and active C57BL/6J mouse skin microbiota, we further attempted to reveal the identity of individual taxa using a nested approach including genus-specific $16 \mathrm{~S}$ rRNA gene primers for subsequent cloning and Sanger sequencing, followed by matching ASV sequences. We recovered 223 sequences from ten wild samples (18-26 per sample) that were selected to maximize the recovery of Staphylococcus ASVs. Due to the overall low skin biomass and comparatively lower Staphylococcus abundance in most laboratory mice, amplification with this primer pair yielded only 25 sequences from 2 laboratory individuals (2 to 23 per sample). In wild individuals, $S$. equorum and $S$. xylosus are preeminent among clone sequences, with comparatively fewer belonging to $S$. cohnii and $S$. succinus. In HL-Lab samples, most clones are $S$. epidermidis, with fewer sequences classifying as $S$. hominis and S. pasteuri (Supplementary Table 4).

Next, to assign species-level taxonomy to the core Staphylococcus ASVs $(\mathrm{n}=33)$, we aligned representative ASV sequences to the Staphylococcus clone sequences obtained above, which yielded matches for all ASVs (Supplementary Table 4). This reveals ASV_1, ASV_3, ASV_4, and ASV_17 to most closely match $S$. xylosus / saprophyticus, ASV_2 and ASV_11 to most closely match S. equorum, and ASV_19 and ASV_77 to most closely match S. epidermidis and $S$. hominis, respectively. Upon inspection of the distribution of Staphylococcus ASVs across mouse groups (Fig. 5a), we observe that wild mice harbor high species diversity, in contrast to C57BL/6J, which contains almost exclusively $S$. xylosus/saprophyticus. Of note, $S$. xylosus/saprophyticus is mostly driven by distinct ASVs in wild and C57BL/6J mice whereby wild individuals harbor ASV_3 and ASV_4 and C57BL/6J show ASV_1 and ASV_17 (Supplementary Fig. 5A). In contrast, same $S$. epidermidis and $S$. hominis ASVs are detected across mouse groups, but remain extremely low in the wild (Supplementary Fig. 5B, C).

To further validate Staphylococcus species patterns, we obtained sequences from a portion of the tuf gene, which provides better resolution for Staphylococcus [66], in a

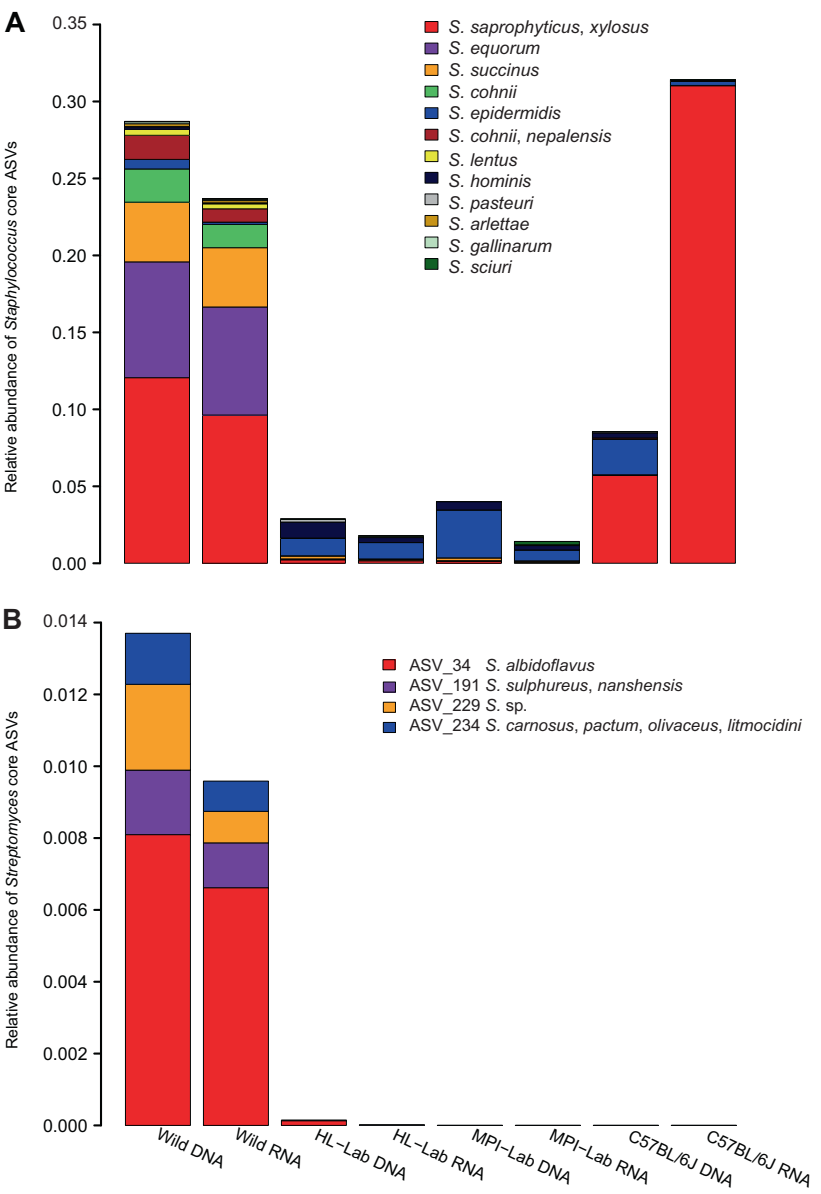

Fig. 5 Relative abundance of Staphylococcus and Streptomyces taxa. Staphylococcus (a) and Streptomyces (b) in standing (DNAbased) and active (RNA-based) communities of wild and laboratory mice. Taxonomy is based on Sanger sequencing of genus-specific $16 \mathrm{~S}$ rRNA gene amplicons.

subset of 73 samples including 49 wild, 11 HL-Lab, 12 MPI-Lab, and 1 C57BL/6J. Overall, we detect 779 Staphylococcus ASVs, of which 442 could be assigned species-level identity. We find 665 ASVs unique to wild mice, 128 of which belong to $S$. xylosus and S. equorum. In contrast, only 50 ASVs are unique to laboratory groups, of which most belong to $S$. hominis and $S$. epidermidis. Interestingly, 64 ASVs are common to wild and laboratory mice, and mostly match $S$. xylosus (9 ASVs), S. equorum (8 ASVs), and S. succinus (6 ASVs) (Supplementary Table 5). Overall, analysis of Staphylococcus based on the tuf gene reveals substantial diversity in the wild, with most ASVs belonging to $S$. equorum and $S$. xylosus. While numerous species are shared between wild and laboratory animals, they are characterized by distinct ASVs, as the fraction of shared ASVs remains minor. Finally, another noteworthy observation is that wild mice display a preponderance of taxa whose closest matches are to the phylogenetically closely related group of novobiocin-resistant species [67], 
which is important in view of the dearth of sequences matching taxa classified as novobiocin-susceptible in wild mice.

Interestingly, novobiocin is also known to be secreted by Streptomyces niveus (S. spheroides) [68], and Streptomyces is similarly a powerful indicator of the wild mice (association statistics 0.91 and $0.86, p \leq 0.05$ in standing and active datasets, respectively). Accordingly, we explored Streptomyces species in wild mice using genus-specific $16 \mathrm{~S}$ rRNA gene primers as described above and recovered 135 clones from the eight samples with the highest diversity of Streptomyces traits (12-20 sequences per sample). This reveals S. albidoflavus (ASV_34) and Sulphureus/nanshensis (ASV_191) to be widespread in the standing and active datasets. S. niveus, the described secretor of novibiocin, is however not detected among clone sequences (Fig. 5b and Supplementary Table 4).

\section{Sources of variation in skin microbiota composition in wild house mice}

To examine potential sources of variation in the composition of wild skin microbiota that may contribute to the community characteristics revealed above, we analyzed environmental and genetic parameters unique to the wild mouse dataset, including geographic sampling location, neutral microsatellite markers, and mitochondrial D-loop sequences. These serve as proxies of the local environment, population structure and maternal transmission, respectively. Analysis of D-loop sequences reveals five distinct haplogroups, whereas analysis of microsatellites reveals $K=13$ as the optimal number of genetic clusters (Supplementary Table 1). Of the 203 wild-caught individuals, 124 are non-admixed ( $\geq 80 \%$ of their ancestry is assigned to a given cluster) and 79 are admixed $(<80 \%$ of their ancestry is assigned to a given cluster) (Supplementary Fig. 6). Using a mixed effect modeling and partial correlation framework to control for the influence of population structure and maternal transmission, we subsequently evaluated the influence of farm characteristics, geographic distance, and host features on community composition in 115 selected individuals.

Among the factors examined, farm or location of sampling is associated with variation in wide-reaching aspects of community composition and structure as expressed by $R^{2} \mathrm{~m}$. In the standing communities (Supplementary Table 6), farm influences the abundance of Bacteroidetes $(77.33 \%$ of total explained variance) and all four beta diversity measures (Bray-Curtis, Jaccard, unweighted, and weighted unifrac), and explains substantial fractions of variance for the Jaccard index (PC1 90.8\% and PC2 88.35\%).

In the active communities, farm and weight influence Staphylococcus and jointly explain $39.38 \%$ of the total variance. Additionally, farm explains important portions of variance in alpha-diversity and beta-diversity measures, and explains up to 74.66 and $63.29 \%$ in the Jaccard index (PC1, and PC2, respectively) (Supplementary Table 7).

In addition to farm and host features, we investigated the influence of geographic distance between sampling locations. Based on the geographic coordinates of sampling sites, we calculated Euclidian pairwise distances for the same 115 individuals mentioned above (geographic coordinates of sampling locations are presented in Supplementary Table 8 and Supplementary Fig. 7). Partial Mantel tests included distances between sampling locations as the main variable, and Cavalli-Soforza and p-distance matrices as conditions to account for correlations between genetic (Cavalli-Soforza, $p$-distance) and geographic distances (See Supplementary Methods) (Mantel test: Sampling locations distances-Cavalli-Soforza, $r=0.23, p=0.0009$; Sampling locations distances-p-distance $r=0.19, \quad p=0.0009$; Cavalli-Soforza-p-distance, $r=0.17, p=0.0009$, based on Spearman's correlation and 1000 permutations). Response variables included all four beta diversity measures in both the standing and active communities. In the standing communities, geographic distance significantly and positively correlates with all four beta diversity measures (Bray-Curtis: $r=0.20, p=0.001$, Jaccard: $r=0.23, p=$ 0.001 , unweighted UniFrac: $r=0.23, p=0.001$, weighted UniFrac: $r=0.13, p=0.001$ ). In the active communities, we find positive significant correlations between sampling locations and all diversity measures (Bray-Curtis: $r=$ $0.068, p=0.008$, Jaccard: $r=0.062, p=0.008$, unweighted Unifrac: $r=0.088, p=0.001$, weighted UniFrac: $r=$ $0.08, p=0.003$ ). Of note, correlation coefficients are substantially lower in the active compared to the standing communities.

In summary, we find that farm characteristics influence a large number of taxa and diversity measures in the standing and active communities, and despite a relatively restricted sampling area, we detect a "distance-decay" similarity pattern in the skin microbiota among wild mice.

\section{Discussion}

The house mouse is an important model for understanding the skin microbiota in health and disease. Moreover, given the marked differences in the immune system of free-living mice versus those living under the "abnormally hygienic" conditions of specific pathogen-free (SPF) barrier facilities [7, 8], characterizing skin microbiota of natural mouse populations is of critical importance, as it offers a more accurate window into the microbial communities with which mice evolved and provides possible explanations for why wild mice more closely resemble human immune traits. As such, our study reports the first description of the native 
skin communities of free-living mice, and contrasts standing and active communities between wild-caught and laboratory mice reared in different facilities, including the C57BL/6J strain typically used in skin biomedical research. We reveal a number of salient features of the wild mouse skin microbiota that may bear relevance on future experimental models. In addition, the contrast in patterns observed between standing and active communities among groups of mice provides valuable insight into the general interpretation of diversity patterns among low biomass skin samples.

\section{Compositional similarities and differences between laboratory and wild habitats}

Despite drastic differences in environmental conditions, and thus in potential colonizing species pools between the laboratory and wild habitats, wild-caught and laboratoryreared mice harbor overall similar communities at the genus level, whereby shared taxa comprise the largest part of the core genera abundance within communities (between $58-78 \%$ and $48-65 \%$ in standing and active communities, respectively). This suggests strong host selection upon the available species pool, whereby the skin is a specialized habitat (e.g., structurally, biochemically) that results in bacterial associations largely independent of the surrounding environment. These observations are akin to those in amphibians, which harbor skin microbiota largely distinct from their aquatic environment [13, 69, 70]. On the other hand, wild mice display significantly higher taxon richness and a higher number of unique taxa than laboratory mice, which is observed in both the standing and active communities. This suggests that these taxa may be true residents and reflect a rare biosphere of unknown functional importance. Compositional differences are also clearly reflected by beta diversity, indicator taxa and random forest analyses, the latter of which identify a number of important candidates such as members of Staphylococcus and Streptomyces.

\section{Staphylococcus taxa closely matching novobiocin- resistant species dominate the skin microbiota of wild house mice}

The observation that Staphylococcus species and ASVs markedly differ between the wild and laboratory environments is potentially of great importance. This genus is one of the most dominant taxa of the human skin microbiota, whose individual members can have a profound impact on health and disease, and we reveal that Staphylococcus abundance is considerably higher in wild mice, with the exception of high Staphylococcus activity (RNA-based abundance) in C57BL/6J. Furthermore, its within-genus composition- defined with a combination of $16 \mathrm{~S}$ rRNA and tuf gene analyses also differs substantially between laboratory and natural populations. Although our study is limited to only metagenomic analysis as samples processed in the field were not prepared for future culture-based analysis and phenotypic screening, we find sequences matching novobiocin-resistant species ( $S$. xylosus, S. equorum, together with $S$. cohnii, S. succinus) to drive Staphylococcus abundance in the wild, while laboratory HL-Lab and MPILab mice harbor sequences matching novobiocinsusceptible $S$. hominis and $S$. epidermidis. Notably, $S$. hominis, and S. epidermidis are detected in the standing wild microbiota, albeit at marginal abundances. Interestingly, however, we reveal that Staphylococcus within the active C57BL/6J skin microbiota is dominated by sequences matching S. xylosus, but with ASVs distinct from the wild.

Given the predominance of sequences matching novobiocin-resistant Staphylococcus species together with Streptomyces being an indicator of wild mice, we investigated whether these observations might be explained by the presence of Streptomyces niveus, a known secretor of novobiocin. However, we did not detect $S$. niveus in wild mice. One possible scenario is that novobiocin-resistant Staphylococcus species are resistant against other antibiotics present in the wild, to which S. epidermis and S. hominis are susceptible. Indeed, Resch et al. [71] and Jeong et al. [72] reported that some $S$. equorum and $S$. xylosus strains are resistant to several antibiotics secreted by members of Actinomycetales, such as chloramphenicol, lincomycin and erythromycin. Of note, $S$. epidermidis strains can be susceptible to erythromycin [73], an antibiotic secreted by Saccharopolyspora erythraea, and Saccharopolyspora is an indicator of wild mice. Thus, other antibiotics potentially present in the wild may lead to a competitive advantage for novobiocin-resistant Staphylococcus species.

To date, little is known about interactions between novobiocin-resistant Staphylococcus and the skin. S. xylosus is described as a skin commensal and potential opportunistic pathogen in human and mouse [74-76]. Interestingly, $S$. xylosus triggered a specific immune response in the mouse skin, similar to $S$. epidermidis, a crucial player in human skin health [77-79]. Furthermore, $S$. equorum strains have been isolated from the skin of animals such as horses and sheep [67], fermented food, and human skin wounds [80]. To date, $S$. equorum has not been described to inhabit the mouse skin. Whether S. equorum and/or $S$. xylosus are functionally equivalent to $S$. epidermis in shaping skin inflammatory and defensive homeostasis requires further exploration.

\section{Environmental influences differentially reflected by DNA- compared to RNA-based profiling}

The mice included in our analyses differ in their environmental origin at a number of levels. First, the laboratory 
mouse groups were housed in distinct facilities that differ in aspects of animal husbandry, which is known to lead to different fecal microbiota composition between individuals of a lab strain common to different facilities [81]. Our analyses identified an unclassified genus belonging to Muribaculaceae, which is a prominent and specific member of the mammalian gut microbiota [82, 83], to be particularly abundant in the standing communities of MPI-Lab mice. This group is comprised of wild-derived individuals that are many generations removed from their wild predecessors. However, due to behavioral differences compared to inbred lab strains, the MPI-Lab mice are maintained with different husbandry routines, including a lower frequency of cage changing (every two compared to one week for the HLLab). Thus, the differential abundance of unclassified Muribacaculaceae (and its phylum Bacteroidetes) at the DNA level in MPI-Lab mice may represent inactive carryover from fecal material. Second, the wild mice being sampled from 34 different farm locations provided a much broader range of environmental differences than within lab facilities, and multivariate analysis yielded a significant influence of farm on numerous aspects of skin community structure. Of note, farm is nearly the only factor found to influence microbial traits in the standing communities, whereas other factors such as weight and BMI are significant for many traits in the active communities. Further, similar to other studies reporting an influence of geography on the composition of skin microbiota of wild mammals [14, 29-31], we observe a pattern of similarity-distance decay, which is stronger in the standing communities. Taken together, the observation of potential non-skinresident taxa and a more prominent influence of environmental variables or their proxies (similarity-distance decay) at the DNA level suggest that RNA-based profiling may reduce environmental noise in studies of the mouse skin microbiota.

In summary, we provide a first description of the skin microbiota of free-living wild mice, which yields important insights on the mouse as a model for biomedical research. Despite overall similarity between lab and wild mice suggesting strong host selection, key aspects of wild mice that appear to be missing in the laboratory environment include Actinobacteria genera, unique Staphylococcus ASVs and many rare, but active taxa. Given the contrasting immune phenotypes observed in wild- compared to laboratory mice $[7,8]$, some of which notably also differ in Staphylococcus composition [6], the differences observed in our study could represent candidates for differential outcome in skin disease models. Thus, future experimental evaluation of these taxa in the mouse skin, as well as the use of wild mice, would be warranted, and we further recommend the use of RNAbased bacterial profiling for the skin when possible.

\section{Data avilability}

Profiles of neutral microsatellite loci are provided in Supplementary Table 9. Mitochondrial D-loop sequences are deposited in GenBank with accession numbers: MN027281-MN027496. Staphylococcus and Streptomyces clone sequences are deposited in GenBank with accession numbers: MN134086-MN134339, and MN161249-MN161392, respectively. 16S rRNA and tuf genes sequences are deposited in the Sequence Read Archive under BioProject PRJNA549583.

Acknowledgements We thank Jan Schubert, Janin Braun, Theresa Wolff, Janice Seidel, and Jana Neckelmann for field assistance, Jan Schubert, Silke Carstensen, Olga Eitel, and Katja CloppenborgSchmidt for excellent technical assistance, and Malte Rühlemann for fruitful discussions, the International Max Planck Research School (IMPRS) and funding by the Deutsche Forschungsgemeinschaft (DFG, German Research Foundation) under Germany's Excellence StrategyEXC 22167-390884018 and by the DFG KFO 303 and CRC 1182.

Author contributions MB and JFB designed the study, MB and MV performed fieldwork and caught and sampled wild mice, MB collected skin samples from laboratory mice, processed samples (nucleic acids extraction), and performed all analyses of the skin microbiota. MV organized the field trip, generated, and carried out mouse genetic analyses. SI provided information and interpretation of the HL-Lab mouse population results. AC contributed to the tuf gene sequencing, and CJC contributed C57BL/6J mouse data. MB and JFB interpreted the results and co-wrote the manuscript.

\section{Compliance with ethical standards}

Conflict of interest The authors declare that they have no conflict of interest.

Publisher's note Springer Nature remains neutral with regard to jurisdictional claims in published maps and institutional affiliations.

Open Access This article is licensed under a Creative Commons Attribution 4.0 International License, which permits use, sharing, adaptation, distribution and reproduction in any medium or format, as long as you give appropriate credit to the original author(s) and the source, provide a link to the Creative Commons license, and indicate if changes were made. The images or other third party material in this article are included in the article's Creative Commons license, unless indicated otherwise in a credit line to the material. If material is not included in the article's Creative Commons license and your intended use is not permitted by statutory regulation or exceeds the permitted use, you will need to obtain permission directly from the copyright holder. To view a copy of this license, visit http://creativecommons. org/licenses/by/4.0/.

\section{References}

1. Ley RE, Peterson DA, Gordon JI. Ecological and evolutionary forces shaping microbial diversity in the human intestine. Cell 2006; 124:837-48.

2. Costello EK, Stagaman K, Dethlefsen L, Bohannan BJ, Relman DA. The application of ecological theory toward an understanding of the human microbiome. Science 2012;336:1255-62. 
3. Shafquat A, Joice R, Simmons SL, Huttenhower C. Functional and phylogenetic assembly of microbial communities in the human microbiome. Trends Microbiol 2014;22:261-6.

4. Koskella B, Hall LJ, Metcalf CJE. The microbiome beyond the horizon of ecological and evolutionary theory. Nat Ecol Evol. 2017;1:1606-15.

5. Zhou J, Ning D. Stochastic community assembly: does it matter in microbial ecology? Microbiol Mol Biol Rev. 2017;81:e00002-17.

6. Rosshart SP, Herz J, Vassallo BG, Hunter A, Wall MK, Badger JH, et al. Laboratory mice born to wild mice have natural microbiota and model human immune responses. Science. 2019;365:eaaw4361.

7. Beura LK, Hamilton SE, Bi K, Schenkel JM, Odumade OA, Casey KA, et al. Normalizing the environment recapitulates adult human immune traits in laboratory mice. Nature 2016;532:512-6.

8. Reese TA, Bi K, Kambal A, Filali-Mouhim A, Beura LK, Burger $\mathrm{MC}$, et al. Sequential infection with common pathogens promotes human-like immune gene expression and altered vaccine response. Cell Host Microbe. 2016;19:713-9.

9. McKenzie VJ, Bowers RM, Fierer N, Knight R, Lauber CL. Cohabiting amphibian species harbor unique skin bacterial communities in wild populations. ISME J. 2012;6:588-96.

10. Kueneman JG, Parfrey LW, Woodhams DC, Archer HM, Knight R, McKenzie VJ. The amphibian skin-associated microbiome across species, space and life history stages. Mol Ecol 2014;23:1238-50.

11. Walke JB, Becker MH, Loftus SC, House LL, Cormier G, Jensen $\mathrm{RV}$, et al. Amphibian skin may select for rare environmental microbes. ISME J. 2014;8:2207-17.

12. Weiland-Brauer N, Neulinger SC, Pinnow N, Kunzel S, Baines JF, Schmitz RA. Composition of bacterial communities associated with aurelia aurita changes with compartment, life stage, and population. Appl Environ Microbiol. 2015;81:6038-52.

13. Jani AJ, Briggs CJ. Host and aquatic environment shape the amphibian skin microbiome but effects on downstream resistance to the pathogen batrachochytrium dendrobatidis are variable. Front Microbiol 2018;9:487.

14. Ross AA, Muller KM, Weese JS, Neufeld JD. Comprehensive skin microbiome analysis reveals the uniqueness of human skin and evidence for phylosymbiosis within the class Mammalia. Proc Natl Acad Sci USA. 2018;115:E5786-95.

15. Brooks AW, Kohl KD, Brucker RM, van Opstal EJ, Bordenstein SR. Phylosymbiosis: relationships and functional effects of microbial communities across host evolutionary history. PLoS Biol 2016;14:e2000225.

16. Federici E, Rossi R, Fidati L, Paracucchi R, Scargetta S, Montalbani E, et al. Characterization of the skin microbiota in italian stream frogs (rana italica) infected and uninfected by a cutaneous parasitic disease. Microbes Environ 2015;30:262-9.

17. Longo AV, Savage AE, Hewson I, Zamudio KR. Seasonal and ontogenetic variation of skin microbial communities and relationships to natural disease dynamics in declining amphibians. $\mathrm{R}$ Soc Open Sci. 2015;2:140377.

18. Walke JB, Becker MH, Loftus SC, House LL, Teotonio TL, Minbiole KP, et al. Community structure and function of amphibian skin microbes: an experiment with bullfrogs exposed to a chytrid fungus. PLoS ONE 2015;10:e0139848.

19. Rebollar EA, Hughey MC, Medina D, Harris RN, Ibanez R, Belden LK. Skin bacterial diversity of Panamanian frogs is associated with host susceptibility and presence of Batrachochytrium dendrobatidis. ISME J 2016;10:1682-95.

20. Cogen AL, Nizet V, Gallo RL. Skin microbiota: a source of disease or defence? Br J Dermatol. 2008;158:442-55.

21. Kostic AD, Howitt MR, Garrett WS. Exploring host-microbiota interactions in animal models and humans. Genes Dev 2013; 27:701-18.

22. Schommer NN, Gallo RL. Structure and function of the human skin microbiome. Trends Microbiol 2013;21:660-8.
23. Myles IA, Williams KW, Reckhow JD, Jammeh ML, Pincus NB, Sastalla I, et al. Transplantation of human skin microbiota in models of atopic dermatitis. JCI Insight. 2016;1:e86955.

24. Linnenbrink M, Wang J, Hardouin EA, Kunzel S, Metzler D, Baines JF. The role of biogeography in shaping diversity of the intestinal microbiota in house mice. Mol Ecol 2013;22:1904-16.

25. Kreisinger J, Cizkova D, Vohanka J, Pialek J. Gastrointestinal microbiota of wild and inbred individuals of two house mouse subspecies assessed using high-throughput parallel pyrosequencing. Mol Ecol 2014;23:5048-60.

26. Wang J, Kalyan S, Steck N, Turner LM, Harr B, Kunzel S, et al. Analysis of intestinal microbiota in hybrid house mice reveals evolutionary divergence in a vertebrate hologenome. Nat Commun 2015;6:6440.

27. Weldon L, Abolins S, Lenzi L, Bourne C, Riley EM, Viney M. The gut microbiota of wild mice. PLoS ONE 2015;10:e0134643.

28. Suzuki TA, Nachman MW. Spatial heterogeneity of gut microbial composition along the gastrointestinal tract in natural populations of house mice. PLoS ONE 2016;11:e0163720.

29. Cheng Y, Fox S, Pemberton D, Hogg C, Papenfuss AT, Belov K. The Tasmanian devil microbiome-implications for conservation and management. Microbiome 2015;3:76.

30. Lavrinienko A, Tukalenko E, Mappes T, Watts PC. Skin and gut microbiomes of a wild mammal respond to different environmental cues. Microbiome 2018;6:209.

31. Avena CV, Parfrey LW, Leff JW, Archer HM, Frick WF, Langwig KE, et al. Deconstructing the bat skin microbiome: influences of the host and the environment. Front Microbiol 2016;7:1753.

32. Grice EA, Kong HH, Renaud G, Young AC, Bouffard GG, Blakesley RW, et al. A diversity profile of the human skin microbiota. Genome Res 2008;18:1043-50.

33. Prager EM, Sage RD, Gyllensten U, Thomas WK, Hübner R, Jones CS, et al. Mitochondrial DNA sequence diversity and the colonization of Scandinavia by house mice from East Holstein. Biol J Linn. 1993;50:85-122.

34. Kearse M, Moir R, Wilson A, Stones-Havas S, Cheung M, Sturrock S, et al. Geneious basic: an integrated and extendable desktop software platform for the organization and analysis of sequence data. Bioinformatics 2012;28:1647-9.

35. Tamura K, Peterson D, Peterson N, Stecher G, Nei M, Kumar S. MEGA5: molecular evolutionary genetics analysis using maximum likelihood, evolutionary distance, and maximum parsimony methods. Mol Biol Evol. 2011;28:2731-9.

36. Huson DH, Bryant D. Application of phylogenetic networks in evolutionary studies. Mol Biol Evol. 2006;23:254-67.

37. Bonhomme F, Orth A, Cucchi T, Rajabi-Maham H, Catalan J, Boursot P, et al. Genetic differentiation of the house mouse around the Mediterranean basin: matrilineal footprints of early and late colonization. Proc Biol Sci. 2011;278:1034-43.

38. Thomas M, Moeller F, Wiehe T, Tautz D. Technical article. A pooling approach to detect signatures of selective sweeps in genome scans using microsatellites. Mol Ecol Notes. 2007;7:400-3.

39. Hardouin EA, Chapuis J-L, Stevens MI, van Vuuren JB, Quillfeldt $\mathrm{P}$, Scavetta RJ, et al. House mouse colonization patterns on the sub-Antarctic Kerguelen Archipelago suggest singular primary invasions and resilience against re-invasion. BMC Evol Biol 2010;10:325.

40. Pritchard JK, Stephens M, Donnelly P. Inference of population structure using multilocus genotype data. Genetics 2000;155: 945-59.

41. Falush D, Stephens M, Pritchard JK. Inference of population structure using multilocus genotype data: linked loci and correlated allele frequencies. Genetics 2003;164:1567-87.

42. Earl DA, vonHoldt BM. STRUCTURE HARVESTER: a website and program for visualizing STRUCTURE output and implementing the Evanno method. Conserv Genet 2011;4:359-61. 
43. Cavalli-Sforza LL, Edwards AWF. Phylogenetic analysis: models and estimation procedures. Evolution 1967;21:550-70.

44. Meirmans PG, Van Tienderen PH. genotype and genodive: two programs for the analysis of genetic diversity of asexual organisms. Mol Ecol Notes. 2004;4:792-4.

45. Callahan BJ, McMurdie PJ, Rosen MJ, Han AW, Johnson AJ, Holmes SP. DADA2: high-resolution sample inference from Illumina amplicon data. Nat Methods 2016;13:581-3.

46. Quast C, Pruesse E, Yilmaz P, Gerken J, Schweer T, Yarza P, et al. The SILVA ribosomal RNA gene database project: improved data processing and web-based tools. Nucleic Acids Res. 2013;41:D590-6.

47. Davis NM, Proctor DM, Holmes SP, Relman DA, Callahan BJ. Simple statistical identification and removal of contaminant sequences in marker-gene and metagenomics data. Microbiome 2018;6:226.

48. Weiss S, Xu ZZ, Peddada S, Amir A, Bittinger K, Gonzalez A, et al. Normalization and microbial differential abundance strategies depend upon data characteristics. Microbiome 2017;5:27.

49. Belheouane M, Gupta Y, Künzel S, Ibrahim S, Baines JF. Improved detection of gene-microbe interactions in the mouse skin microbiota using high-resolution QTL mapping of 16S rRNA transcripts. Microbiome 2017;5:59.

50. Benjamini Y, Hochberg Y. Controlling the false discovery rate a practical and powerful approach to multiple testing. J R Stat. 1995;57:289-300.

51. De Caceres M, Legendre P. Associations between species and groups of sites: indices and statistical inference. Ecology 2009; 90:3566-74.

52. De Cáceres $M$, Legendre $P$, Moretti $M$. Improving indicator species analysis by combining groups of sites. Oikos 2010;119: 1674-84.

53. Wiener M, Liaw A. Classification and regression by randomForest. Newsl R Project. 2002;2:18-22.

54. Wang Q, Garrity GM, Tiedje JM, Cole JR. Naive Bayesian classifier for rapid assignment of rRNA sequences into the new bacterial taxonomy. Appl Environ Microbiol. 2007;73:5261-7.

55. McMurray CL, Hardy KJ, Calus ST, Loman NJ, Hawkey PM. Staphylococcal species heterogeneity in the nasal microbiome following antibiotic prophylaxis revealed by tuf gene deep sequencing. Microbiome 2016;4:63.

56. Lamers RP, Muthukrishnan G, Castoe TA, Tafur S, Cole AM, Parkinson CL. Phylogenetic relationships among Staphylococcus species and refinement of cluster groups based on multilocus data. BMC Evol Biol. 2012;12:171.

57. Oksanen J, Blanchet FG, Kindt R, Legendre P, Minchin PR, O'Hara RB, et al. Vegan: community ecology package. R Package Version 22-0. 2015.

58. Wright ES. Using DECIPHER v2.0 to analyze big biological sequence data in R. R J. 2016.

59. Schliep KP. phangorn: phylogenetic analysis in R. Bioinformatics 2011;27:592-3.

60. Faith DP. Phylogenetic pattern and the quantification of organismal biodiversity. Philos Trans R Soc Lond B. 1994;345:45-58.

61. Kembel SW, Cowan PD, Helmus MR, Cornwell WK, Morlon H, Ackerly DD, et al. Picante: R tools for integrating phylogenies and ecology. Bioinformatics 2010;26:1463-4.

62. Hamady M, Lozupone C, Knight R. Fast UniFrac: facilitating high-throughput phylogenetic analyses of microbial communities including analysis of pyrosequencing and PhyloChip data. ISME J. 2010;4:17-27.

63. Lozupone C, Lladser ME, Knights D, Stombaugh J, Knight R. UniFrac: an effective distance metric for microbial community comparison. ISME J. 2011;5:169-72.
64. McMurdie PJ, Holmes S. phyloseq: an R package for reproducible interactive analysis and graphics of microbiome census data. PLoS ONE 2013;8:e61217.

65. Bates D, Mächler M, Bolker B, Walker S. Fitting linear mixedeffects models using lme4. J Stat Softw. 2015;67:1-48.

66. Hwang SM, Kim MS, Park KU, Song J, Kim EC. tuf gene sequence analysis has greater discriminatory power than $16 \mathrm{~S}$ rRNA sequence analysis in identification of clinical isolates of coagulase-negative staphylococci. J Clin Microbiol. 2011;49:4142-9.

67. Becker K, Heilmann C, Peters G. Coagulase-negative staphylococci. Clin Microbiol Rev. 2014;27:870-926.

68. Steffensky M, Muhlenweg A, Wang ZX, Li SM, Heide L. Identification of the novobiocin biosynthetic gene cluster of Streptomyces spheroides NCIB 11891. Antimicrob Agents Chemother. 2000;44:1214-22.

69. Fitzpatrick BM, Allison AL. Similarity and differentiation between bacteria associated with skin of salamanders (Plethodon jordani) and free-living assemblages. FEMS Microbiol Ecol. 2014;88:482-94.

70. Loudon AH, Woodhams DC, Parfrey LW, Archer H, Knight R, McKenzie V, et al. Microbial community dynamics and effect of environmental microbial reservoirs on red-backed salamanders (Plethodon cinereus). ISME J 2014;8:830-40.

71. Resch M, Nagel V, Hertel C. Antibiotic resistance of coagulasenegative staphylococci associated with food and used in starter cultures. Int J Food Microbiol. 2008;127:99-104.

72. Jeong D-W, Heo S, Ryu S, Blom J, Lee J-H. Genomic insights into the virulence and salt tolerance of Staphylococcus equorum. Sci Rep. 2017;7:5383.

73. Lin MH, He L, Gao J, Liu YX, Suo JJ, Xing YB, et al. Penetration of erythromycin through Staphylococcus epidermidis biofilm. Chin Med J 2013;126:2647-51.

74. Nagase N, Sasaki A, Yamashita K, Shimizu A, Wakita Y, Kitai S, et al. Isolation and species distribution of staphylococci from animal and human skin. J Vet Med Sci. 2002;64:245-50.

75. Russo M, Invernizzi A, Gobbi A, Radaelli E. Diffuse scaling dermatitis in an athymic nude mouse. Vet Pathol 2013;50:722-6.

76. Kim Y, Lee Y-S, Yang J-Y, Lee S-H, Park Y-Y, Kweon M-N. The resident pathobiont Staphylococcus xylosus in Nfkbiz-deficient skin accelerates spontaneous skin inflammation. Sci Rep. 2017;7:6348.

77. Naik S, Bouladoux N, Linehan JL, Han S-J, Harrison OJ, Wilhelm $\mathrm{C}$, et al. Commensal-dendritic-cell interaction specifies a unique protective skin immune signature. Nature 2015;520:104-8.

78. Nakamizo S, Egawa G, Honda T, Nakajima S, Belkaid Y, Kabashima K. Commensal bacteria and cutaneous immunity. Semin Immunopathol 2015;37:73-80.

79. Nakatsuji T, Chen TH, Butcher AM, Trzoss LL, Nam SJ, Shirakawa $\mathrm{KT}$, et al. A commensal strain of Staphylococcus epidermidis protects against skin neoplasia. Sci Adv 2018;4:eaao4502.

80. Novakova D, Sedlacek I, Pantucek R, Stetina V, Svec P, Petras P. Staphylococcus equorum and Staphylococcus succinus isolated from human clinical specimens. J Med Microbiol. 2006;55:523-8.

81. Rausch P, Basic M, Batra A, Bischoff SC, Blaut M, Clavel T, et al. Analysis of factors contributing to variation in the C57BL/6J fecal microbiota across German animal facilities. Int $\mathrm{J}$ Med Microbiol. 2016;306:343-55.

82. Ormerod KL, Wood DL, Lachner N, Gellatly SL, Daly JN, Parsons JD, et al. Genomic characterization of the uncultured Bacteroidales family S24-7 inhabiting the guts of homeothermic animals. Microbiome 2016;4:36.

83. Lagkouvardos I, Lesker TR, Hitch TCA, Gálvez EJC, Smit N, Neuhaus K. Sequence and cultivation study of Muribaculaceae reveals novel species, host preference, and functional potential of this yet undescribed family. Microbiome. 2019;7:28. 